\title{
TESTING THE ROBUSTNESS OF CIRCULARITY INDICATORS: EMPIRICAL INSIGHTS FROM WORKSHOPS ON AN INDUSTRIAL PRODUCT
}

\author{
Saidani, Michael; Cluzel, François; Leroy, Yann; Yannou, Bernard \\ Laboratoire Genie Industriel, CentraleSupélec, Université Paris-Saclay
}

\begin{abstract}
Monitoring properly the circularity performance of technical products is a point of increasing importance. Yet, evaluating the circularity potential of products during (re)design and development phases is a challenging task. In this study, several C-indicators are experienced by doctoral students and industrialists through two workshops on a real-world industrial product. The values obtained for each indicator are collected and analyzed: as all participant are working on the same technical product with the same dataset, the circularity scores calculated are compared to discuss the reliability and the uncertainty related to these indicators. These new empirical insights are put in parallel with the existing critical analyses of $\mathrm{C}$-indicators from literature. As a result, future research directions on circularity indicators are advanced and discussed, including: the integration of uncertainty considerations into the assessment methodology of circularity indicators; the uptake by industry of such indicators during product design and development; the link between circularity and sustainability scores.
\end{abstract}

Keywords: Circular economy, Circularity indicators, Design for X (DfX), Case study, Workshop

Contact:

Saidani, Michael

CentraleSupélec, Université Paris-Saclay

Laboratoire Génie Industriel

France

michael.saidani@centralesupelec.fr

Cite this article: Saidani, M., Cluzel, F., Leroy, Y., Yannou, B. (2019) 'Testing the Robustness of Circularity Indicators: Empirical Insights from Workshops on an Industrial Product', in Proceedings of the 22nd International Conference on Engineering Design (ICED19), Delft, The Netherlands, 5-8 August 2019. DOI:10.1017/dsi.2019.347 


\section{INTRODUCTION: CONTEXT AND OBJECTIVES}

Moving towards circular design practices is increasingly encouraged in a context of sustainable development (Geissdoerfer et al., 2017). Design for circularity is actually an emerging field that needs new integrated frameworks, including methods, tools and indicators, to help establishing and monitoring innovative solutions across sectors in line with the circular economy (CE) paradigm (Earley, 2017). In fact, the CE has a dynamic nature that is difficult to assess and monitor but interesting to study through indicators (Bonet et al., 2014). Following a common agreement - shared by academics, industrialists and politics - on the need to provide circular economy related indicators at different systemic levels to facilitate and catalyze this transition, many circularity tools, metrics, and indicators have been developed in the last few years (Evans and Bocken, 2013; EMF, 2015; EASAC, 2016; EEA, 2016; Cayzer et al., 2017; Saidani et al., 2017a). Yet, without a stabilized definition of what circular economy is, it is of utmost importance to know what available circularity indicators measure in order to use them properly. Circularity indicators (C-indicators) can be defined here as a quantitative or qualitative factor or variable that provides a simple and reliable means (OECD, 2014) to assess and monitor the performance of systems in a circular economy perspective. Recently, 55 sets of C-indicators - created and used by scholars, consulting companies and governmental agencies worldwide - have been reviewed and classified into a need-based taxonomy, including 10 categories, driven by the usage of such indicators (Saidani et al., 2019). Parchomenko et al. (2019) also reviewed $63 \mathrm{C}$-indicators, identifying three main clusters of C-indicators, namely: resource efficiency; materials stocks and flows; product-centric.

In this paper, the focus is made on the circularity performance of industrial products and related Cindicators, i.e. at the micro scale of the CE implementation. While Linder et al. (2017) or Saidani et al. (2017b) provided a first qualitative comparison and critical analysis on several product circularity indicators, the present research work addresses more quantitatively the robustness of such indicators, i.e. the variability of circularity scores on a given product. To do so, the results of two workshops experimenting four $\mathrm{C}$-indicators on an industrial case study are analyzed, compared and discussed. The objectives of the two workshops, conducted in 2017 and 2018 during the Spring School EcoSD "Eco-design of complex system" endorsed by the Design Society, are to:

- Introduce the challenges of measuring the circularity performance of industrial products through C-indicators;

- Test the $\mathrm{C}$-indicators Advisor tool and receive feedback from industrial and academic participants;

- Compare the variability in the circularity scores obtained from one group to another, working on the same industrial product;

- Examine further $\mathrm{C}$-indicators and ask participants to make a critical analysis regarding their compliance with the CE paradigm, their robustness, applicability in industry, and userfriendliness.

Additionally, through the industrial case study, participants question how C-indicators can contribute in designing and developing more circular products and systems. In other words, we discuss to what extent these $\mathrm{C}$-indicators provide guidance to enhance the circularity potential of products during the (re)design and development process. Results and insights provided by the participants are reported, summarized and discussed. Their comments and feedbacks are notably put into perspective with: (i) our prior critical analysis (to complement, validate or invalidate it) on C-indicators (Saidani et al., 2017b); (ii) critical reviews on some C-indicators found in the scientific literature (e.g., Linder et al., 2017). The limitations of the interpretations made from these two workshops are then discussed. On this basis, recommendations for future works in developing and implementing $\mathrm{C}$-indicators are made. Last but not least, the findings reveal different possible contributions, from the use of C-indicators in diverse contexts, to catalyze the shift to a more CE. In this line, we detail and provide all the elements to adapt and (re)use this workshop: whether in an industrial environment (e.g., to train industrial practitioners (designers, engineers, or managers) on how they can assess and enhance the circularity performance of their products), or for education purposes in a training sequence (e.g., to introduce the circular economy or more specifically to educate the professional of tomorrow in designing more circular products, services and systems). 


\section{METHODOLOGY}

\subsection{Research approach}

As illustrated and applied in a workshop reported by Leroy et al. (2015) comparing the environmental evaluation of ideas in the early phases of the design process from several groups, the Design Research Methodology (Blessing and Chakrabarti, 2009) recommends to use one control group has a comparative baseline. Reference results provided by our first experimentation of these $\mathrm{C}$-indicators are considered under the "control group", arguing the authors have a more precise and enhanced knowledge of the product and its eco-system, as well as more time to experiment the indicators. Also, to compare in a consistent manner the results and comments brought by the two workshops with prior studies and critical analysis on C-indicators, the same industrial product (here, a catalytic converter) is used.

The Circular Economy Toolkit (CET) (Evans and Bocken, 2013), Material Circularity Indicator (MCI) (EMF, 2015), Circular Economy Indicator Prototype (CEIP) (Cayzer et al., 2017), and Circularity Potential Indicator (CPI) (Saidani et al., 2017a) are the four circularity indicators tested. In addition to the fact these indicators were recommended by the C-Indicators Advisor tool (see sub-section 3.1), we pre-selected these four indicators because they are all coming with an open access computer-based assessment tool (whether a dynamic spreadsheet or a web tool) which makes the calculations more manageable, whereas other potentially relevant indicators are not directly or freely accessible, or are only embodied in a textual format.

Additionally, Leroy et al. (2015) recommend that each group should experience two cases in order to be able to compare them. As such, each group test two different circularity indicators on the same industrial example of a company willing to measure and improve the circularity performance of a product that they designed and developed. The information related to this case study and provided to the participants are synthesized in a 2-page datasheet, including: the industrial context, a simplified version of the catalytic converter with information on its pre-life (e.g., simplified bill of materials, logistics), usage (e.g., lifespan, location) and end-of-life (e.g., collection rate, recycling efficiency). Against this background, participants are surveyed about each $\mathrm{C}$-indicator they experiment, using these questions:

- Do the C-indicator tested encompass the whole complexity of the CE paradigm?

- What are the missing points to an enhanced measurement of product circularity?

- Is the tool relevant for industrial practitioners willing to improve the circularity performance of their products during the (re-)design and development phases? Why?

- Do you see another suitable potential use(s) of this tool? If yes, for what purposes?

- Other comments (e.g., related to the format, utility, areas for improvement) on the $\mathrm{C}$-indicator.

\subsection{Workshop design}

For reasons of time constraints, regarding the planning and organisation of the Spring School EcoSD, in which the workshops have been conducted in 2017 and 2018, the entire session has to fit in half a day (i.e., in three hours). The precise timeline of each activity performed during the workshop is detailed in Table 1. After a short ice-breaking activity, the challenges of assessing the circularity performance at different systemic levels are introduced and the case study is presented. Then, based on the industrial needs specific to the case study, the participants, by groups, use the C-Indicators Advisor tool to identify suitable C-indicators, and two of these indicators are experimented by each group. Eventually, they question the strengths, complementarity and weaknesses of each approach in the light of circular economy, and in response to industrial practitioners willing to evaluate and improve the circularity performance of their products. Importantly, the workshop has been designed so that the participants have the time to fill out the responses documents completely in order to collect their feedbacks and constructive criticisms on the C-indicators they test.

In the first session (in 2017), the 10 participants were splitted into two groups of three and two groups of two. In the second session (in 2018), the 14 participants were splitted into two groups of four and two groups of three. The group distribution is made to be equivalent (regarding the number of $\mathrm{PhD}$ students and industrialists in each groups, when possible) Note that while the evaluation of the circularity performance (i.e., the circularity score of the industrial product) was obtained per group for each C-indicator, the feedbacks of participants were individual. All in all, two workshops were performed for a total of 24 attendees (mainly $\mathrm{PhD}$ students conducting their research in France, but 
also $2 \mathrm{PhD}$ students coming for European universities, 2 assistant professors, and 4 industrialists). During the first session, only two already knew some of the indicators and only one had experienced them before. During the second, only one already knew two of the indicators but had never experienced them.

Table 1. Timeline of the workshop on C-indicators

\begin{tabular}{|c|c|c|c|c|c|}
\hline Group & $45 \mathrm{~min}$ & $45 \mathrm{~min}$ & $30 \mathrm{~min}$ & $30 \mathrm{~min}$ & $30 \mathrm{~min}$ \\
\hline$\# 1$ & \multirow{4}{*}{$\begin{array}{l}\text { Ice-breaking } \\
\text { activity. } \\
\text { Introduction to C- } \\
\text { indicators. }\end{array}$} & \multirow{4}{*}{$\begin{array}{l}\text { Experimentation of the } \\
\text { C-Indicators Advisor tool. } \\
\text { Selection of appropriate } \\
\text { circularity indicators. }\end{array}$} & MCI & CET & \multirow{4}{*}{$\begin{array}{l}\text { Comparison of } \\
\text { the results and } \\
\text { discussion. }\end{array}$} \\
\hline$\# 2$ & & & CEIP & CPI & \\
\hline$\# 3$ & & & CPI & MCI & \\
\hline$\# 4$ & & & CET & CEIP & \\
\hline
\end{tabular}

All the resources and further details regarding the organisation of the workshop are available on demand or in Saidani's PhD thesis (2018), including: an ice-breaking activity, the datasheet related to the industrial case study, and the responses documents. For instance, here is the description of the proposed ice-breaking activity: At the beginning of the workshop, participants are asked to share their current knowledge related to the following questions on an individual answer sheet: What is your definition of a circular economy? Can you cite one or two example(s) of circular practices? What should consider a circularity indicator? Do you know some C-indicators? If so, which one(s)? Such an ice-breaking activity aims to involve the participation of every attendees, making them think on these challenges. It also enables to provide immediate feedback, allowing them to position their current knowledge related to the $\mathrm{CE}$ and $\mathrm{C}$-indicators, as answers are collected and read to everyone (anonymously).

\section{RESULTS AND DISCUSSION}

\subsection{Test of the C-Indicators Advisor tool}

The C-Indicators Advisor tool (Saidani et al., 2019) is first used to identify the most suitable indicators according to the specificities of the case study. This tool has been developed between 2017 and 2018 (i.e., between the two workshops), so it has been experimented for the first time during the second session of this workshop in 2018. Table 2 compares how the requirements provided by the industrialist of this case study are interpreted and translated in inputs of the tool to identify the a priori most appropriate C-indicators. Note that the query tool is designed so that when a criteria input is left blank $(-)$, no filter is applied, and it offers therefore a wider variety of C-indicators in output.

Table 2 - Results from the experimentation of the C-Indicators Advisor

\begin{tabular}{|c|c|c|c|c|c|}
\hline Input (below) & Control & Group \#1-18 & Group \#2-18 & Group \#3-18 & Group \#4-18 \\
\hline Level & Micro & Micro & Micro & Micro & Micro \\
\hline Perspective & - & Potential & Actual & Potential & - \\
\hline Performance & Intrinsic & Intrinsic & Intrinsic & - & - \\
\hline Loop(s) & All the loops & All the loops & All the loops & All the loops & All the loops \\
\hline Dimensionality & - & - & - & - & - \\
\hline Transversality & - & - & - & - & Generic \\
\hline Usages & - & - & - & Decision & - \\
\hline $\begin{array}{l}\text { Type and } \\
\text { format }\end{array}$ & - & - & - & - & - \\
\hline $\begin{array}{l}\text { Output } \\
\text { (C-indicators } \\
\text { advised by } \\
\text { the tool) }\end{array}$ & $\begin{array}{l}\text { 8: CEIP, CET, } \\
\text { CPI, CLC, } \\
\text { MCI, BCI, } \\
\text { IOBS, RDI }^{1}\end{array}$ & $\begin{array}{l}\text { 5: CEIP, } \\
\text { CET, CPI, } \\
\text { CLC, RDI }\end{array}$ & 2: MCI, IOBS & $\begin{array}{l}\text { 5: CEIP, } \\
\text { CET, CPI, } \\
\text { CLC, RDI }\end{array}$ & $\begin{array}{l}\text { 6: CEIP, } \\
\text { CET, CPI, } \\
\text { MCI, IOBS, } \\
\text { RDI }\end{array}$ \\
\hline
\end{tabular}

Globally, the industrialist's (here, a project manager) requirements are well understood and easily translated into specific inputs that fit his needs. As the focus is on an industrial product and associated key components/materials, the micro level of CE implementation is selected. Also, the project

1 Building Circularity Indicators (BCI); Circular Economy Indicator Prototype (CEIP); Circular Economy Toolkit (CET); Closed Loop Calculator (CLC); Circularity Potential Indicator (CPI); Input-Output Balance Sheet (IOBS); Material Circularity Indicator (MCI); Resource Duration Indicator (RDI). 
manager mentioned that he is more interested in the impacts of future designs and possible business models changes on the performance of the inherent (i.e., intrinsic) circularity of his product, and that he wants to consider all possible loops of the CE. Note that no clear information was provided regarding the perspective level of measurement (i.e., between a potential or actual circularity), which leads to different interpretations among the groups. Otherwise, Groups \#1-18, \#3-18 and \#4-18 converge rapidly towards the same indicators set. After reading the complementary information provided in output of the tool for each $\mathrm{C}$-indicator advised, the participants were asked which indicator(s) they would particularly recommend to use and for which reason(s). Group \#1-18 and Group \#3-18 recommended the CPI for the following reasons: "it corresponds well to the need of the project manager", "it seems complete", "to evaluate the circularity potential of a product", "spreadsheet already configured". Group \#2-18 and Group \#4-18 advised the MCI for the following reasons: "it is adaptable to materials, products, and company", "it is an Excel-based tool", "to evaluate the circularity performance of materials". Group \#3-18 additionally prescribed the RDI because it is "good for decision-making".

All participants were also asked if they had in mind other criteria that could be useful to refine further the selection of C-indicators. While the current criteria were sufficient enough for Group \#2-18 and Group \#4-18, Group \#1-18 would have appreciated an indication about the "user-friendliness" on how to calculate the indicator, and the availability of an example of application. To Group \#3-18, the circularity strategies proposed under the "loop(s)" criteria are not exhaustive. Eventually, participants had the possibility to comment freely about the relevance of the C-Indicators Advisor tool (e.g., for a specific usage by industrialists or academics) and on their user experience (e.g., about the utility and usability of the tool). A participant valued it was "very interesting to reduce and select the number of indicators". Another one, who "had no experience on C-indicators", reported "it seems to be a useful tool to know which indicators exist and what are the differences between them", and highlighted the "clear interface" of the tool. Regarding the areas for improvement, one mentioned it misses the open access feature (stating that "using an Excel spreadsheet is not really open access") and the integration of the data to other tools used during the design and development phases of a technical product. Note that to answer this last point, a web-platform is currently under development to use the tool directly online.

\subsection{Experimentation of four C-indicators: CEIP, CET, CPI, MCI}

\subsubsection{Quantitative variability in the results}

The quantitative comparison of the circularity scores obtained by the different groups - all working on the same product and using the same dataset - shows a higher variability in the results of $\mathrm{C}$-indicators assessing a circularity potential (the CET, CEIP, CPI) than of the MCI which evaluates an actual (effective) circularity, as illustrated in Table 3 and Figures $1,2 \& 3$. In fact, the average value of the aggregated MCI is 0.48 (out of 1 ) with a standard deviation below 0.01 ; the average CEIP score has a value of 35.2 (out of 152) with a standard deviation equal to 17.4; the average CPI score has a value of 29.1 (out of 100) with a standard deviation equal to 11.9. Beyond the absolute values of these uncertainties, the relative values between these criteria can be used to prescribe specific actions and oriente decision-making during design and development phases in order to improve the potential performance of circularity. In practice, designers, engineers and decision makers should focus on the areas - embodied here through the sub-criteria of each circularity score - assigned with the lowest circularity scores.

As an illustration, although a variability between groups' results is noticed in the improvement potential assessed by the CET, and in the circularity scores of the CEIP and CPI, they highlight locally and to a certain extent - similar areas of improvement to for an enhanced circularity performance of the catalytic converter. For instance, both the CPI and the CET emphasize a higher room for improvement in the business model and reverse cycles (e.g. considering the refurbishing of the catalytic converter) compared to product design. Advancing such recommendations is less straightforward for the results obtained with the CEIP. Yet, a consensus is preferable to oriente and validate appropriate actions aiming at improving the circularity potential of newly or re-design products. Further explanations of the variability in the results are discussed in the next sub-section. Also, the qualitative analysis of participants' feedbacks illuminates other interesting possible usages and areas for improvement of these $\mathrm{C}$-indicators. 
Table 3 - Results from the experimentation of the Circular Economy Toolkit

\begin{tabular}{llllll}
\hline Improvement Potential & Control & Group \#4-17 & Group \#1-17 & Group \#4-18 & Group \#1-18 \\
\hline Reduce Material & Medium & Medium & Low & Medium & Medium \\
\hline Optimise Materials & High & High & Medium & High & High \\
\hline Industrial Symbiosis & Medium & Low & Medium & Medium & Low \\
\hline Design & None & None & Low & None & None \\
\hline Usage & Medium & Low & Medium & Medium & Low \\
\hline Maintain & Medium & High & Medium & Medium & High \\
\hline Reuse & Medium & Medium & Medium & Medium & Medium \\
\hline Refurbish & High & High & Medium & Medium & Medium \\
\hline Recycle & Medium & Medium & Medium & Medium & Medium \\
\hline Product as a Service & Low & Medium & Medium & Medium & High \\
\hline
\end{tabular}

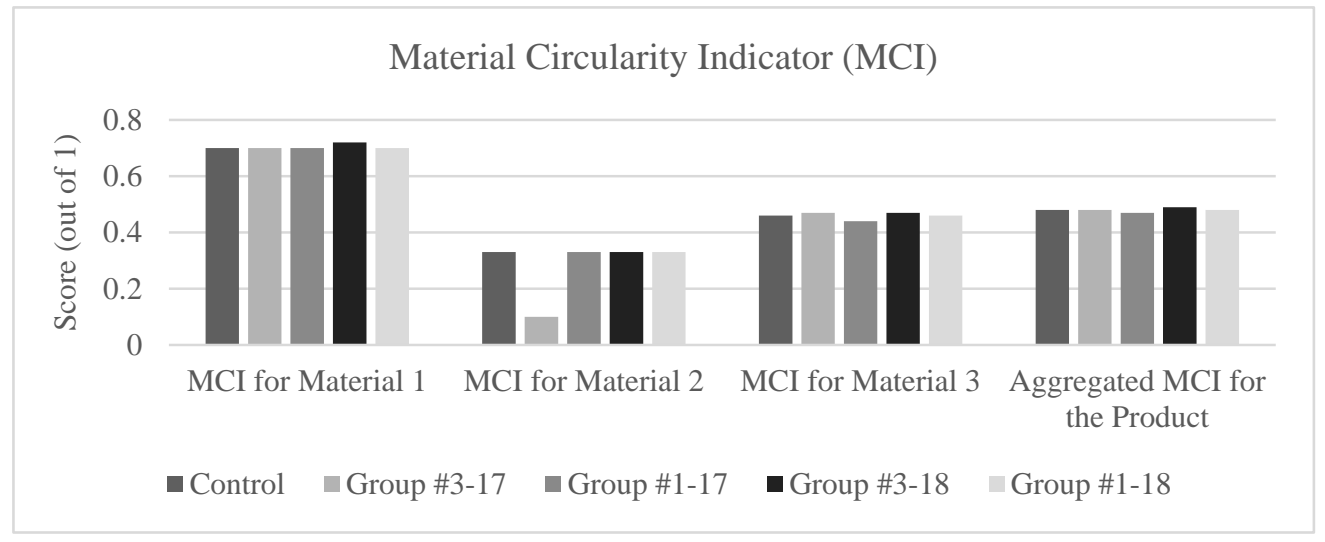

Figure 1. Results from the experimentation of the Material Circularity Indicator

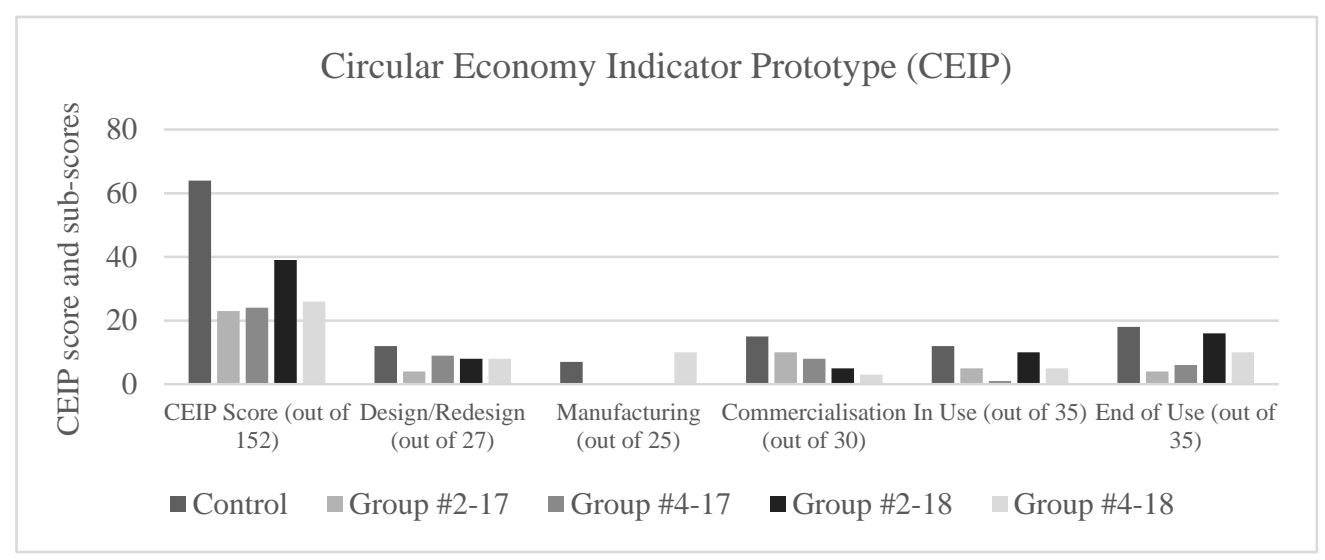

Figure 2. Results from the experimentation of the Circular Economy Indicator Prototype

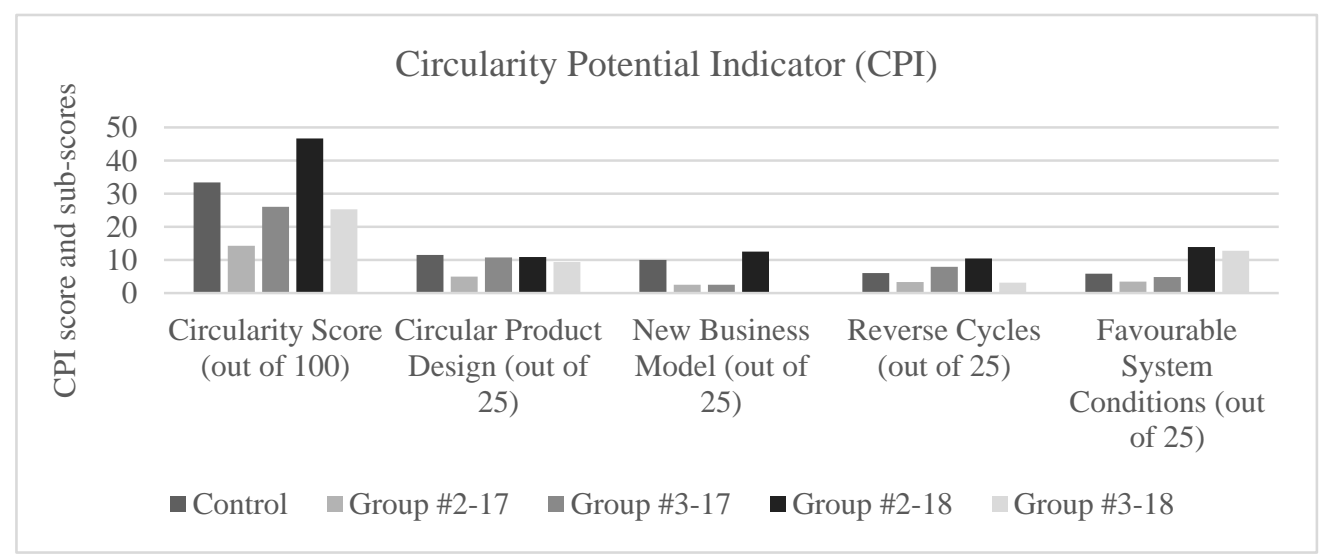

Figure 3. Results from the experimentation of the Circularity Potential Indicator 


\subsubsection{Qualitative feedback and critical analysis}

\subsubsection{Improvement areas}

Regarding a possible explanation about the variability in the circularity scores between each group working on the same case study, almost all the participants mentioned an important uncertainty when answering a question asked by the CEIP, CET or CPI. They stated the reasons of this uncertainty are based both on the lack of clarity of certain questions, and on their lack of knowledge on the technical product and its associated ecosystem. In fact, some possible answers are subjective to the interpretation and background of the user (e.g., "easy", "hard", "cheap", "expensive" in the CET, or "very", "few" in the CPI), which can explain the aforementioned variability in the results. On the other hand, most participants valued not only the user-friendliness (i.e., the tools are easy to use and it is straightforward to understand how they work) but also the time-efficiency (once the data are available and properly collected) of the four tools allowing a practical computation of the circularity scores.

Regarding the missing points and elements these indicators should consider for an enhanced measurement of the product circularity performance, the following comments and suggestions were made. Several participants noticed the economic aspects (e.g., the costs of logistics and end-of-life treatment compared to the inherent value of materials) are not directly taken into consideration by the C-indicators they experienced. Specifically related to the MCI, the comparison between the cost of recycling operations, plus the value of secondary materials, against the cost of virgin material production, plus the value of primary materials, is reported as a missing point. One participant suggested to connect the separate spreadsheets of the MCI (i.e., one file at material level and one file at the product level) into one single tool. More generally, the location and transportation of the product, as well as the business models associated the product are also some points barely addressed by these $\mathrm{C}$-indicators that should have deserved more attention according to some participants. These missing points are related to dynamic evolutions of flows (i.e., cost fluctuations, transportation, etc.) in the circular economy system considered. Capturing additional temporal and spacial elements could improve the relevance of the results provided by these assessment tools. Eventually, one participant would have appreciated to be informed about the role and responsibilities of players involved in each activity that affects the circularity performance, while another one would have valued the fact the Cindicators explicitly display the equation(s) or mathematical calculation(s) to get the circularity scores (e.g., the weighting factors used for computing the CPI).

Participants were also asked if the C-indicators they tested cover the whole complexity of the circular economy. For the four indicators experimented, there was no unambiguous consensus among the participants regarding this question. It is therefore difficult to draw meaningful conclusions regarding the compliance between what is assessed by the $\mathrm{C}$-indicators and the circular economy paradigm. After discussion with some participants, one explanation of their different answers lies on the fact these concepts (complexity of the circular economy, system thinking, lifecycle thinking) are not so well-defined to them, and thereby lead to several subjective interpretations. In this line, Eyckmans et al. (2018) highlight the complexity of determining a credible and usable indicator for the circular economy, notably because the circular economy does not possess an unambiguous definition, and current recycling indicators as metrics of circular economy activity are methodologically unsatisfactory regarding the scope of the circular economy concept.

\subsubsection{Potential uses}

Regarding the relevance of $\mathrm{C}$-indicators and their associated measurement tools for industrial practitioners willing to improve the potential circularity performance of their products during (re)design and development phases, participants provided comments on each C-indicator as it follows. The CET is mainly viewed as useful both for comparison between products, and for quickly identifying where improvements can be made. One also mentioned that to be really relevant, the CET has to be used by experts that know the product deeply in order to answer all the questions accurately. The MCI is recognized as a practical and simple tool for a rapid analysis of a product, providing an early evaluation on material circularity, as well as to be used as a first step in the circularity assessment before digging more deeply into improvement areas. The CEIP appears also relevant for a quick comparison of design strategies, checking the main aspects of circularity. Yet, for some, the level of details required in input data is rather high, while further considerations on business models and marketing aspects are missing in the list of questions asked to compute the CEIP. To most 
participants, the CPI seems useful as a comprehensive checklist, to improve the circularity performance of products, covering design for product circularity, looking at policy and taking into account the business models. Globally, the participants also discussed the genericity of these tools. To some, it is a true advantage that they all can be applied on a wide variety of products. To others, it is an obstacle to go further in the actual improvement of the products, by considering specific and professional knowledge related to the product and its ecosystem.

Regarding other potential suitable use(s) of these C-indicators and associated tools, participants saw promising applications of $\mathrm{C}$-indicators in various contexts. Interestingly, many participants said such indicators could have benefits beyond the pure assessment of the product circularity performance, both in education and industry. For instance, for educational purposes, by being applied on a real-world case study, these $\mathrm{C}$-indicators could be used to introduce in a concrete way the many different facets of the circular economy. More generally, such a hands-on workshop on C-indicators can train current industrial practitioners (e.g., designers, engineers, managers) or the professional of tomorrow to think concretely and critically on how to design and develop more circular systems, as well as to have the tools to pilot industrial and managerial activities in a circular economy perspective.

\subsubsection{Comparison with literature}

Most of these comments are in agreement and complementary with the research carried out by Griffiths and Cayzer (2016) interviewing circular economy players about the CEIP, who mentioned the tool: "is easy to follow", could be used "as a training exercise for engineers", "as a checklist", "to understand the levers for working on circularization", but "is best suited for incremental changes", could be extended to "a comparison of 2-3 product versions on one page". The insights provided by the two workshops are also supplementary to the analyses made by Linder et al. (2017), whose paper on product circularity metrics states that one avenue for future research, related to the adoption of Cindicators in industrial practices, is to examine how collaborative research can contribute to the further development, testing, and implementation of robust and legitimate C-indicators. Linder et al. (2017) particularly discuss the construct validity, reliability, transparency, generality and aggregation principles of five circularity indicators, including the MCI (EMF, 2015). According to them, circular metrics of low dimensionality, such as the MCI, are useful for managerial decision making but their operationalization may appear to be problematic. Additionally, they found that many of the data inputs required might be uncertain or depend on several factors or assumptions, such as the destination of a product after use or the efficiency of recycling processes. They also state that a fully functioning circular economy metric should be able to examine the relationship between product circularity and other variables, such as cost savings (Linder et al., 2017).

\subsection{Limitations}

So far, the workshops have been conducted two times with a total of 24 attendees, mainly from academia. As such, readers should not generalize the results because the values obtained and criticisms made by industrial practitioners may differ. For instance, we discussed if the $\mathrm{C}$-indicators and their associated measurement tools are relevant for supporting industrial practices based on the insights provided by the participants. Even if they provided constructive feedbacks based on their current projects with industry or previous industrial experiences, further experimentations of these Cindicators in an industrial environment are required to demonstrate their actual and effective relevance. Also, post-workshop interviews can be relevant to validate the findings and exchange the viewpoints of one participant to one another. Yet, combining the previous discussions on circularity indicators (e.g., EASAC, 2016; Cayzer et al., 2017; Linder et al., 2017) and the new insights provided by these two workshops, we argue the use of C-indicators can not only enable the integration of circular thinking into the product design and development process, but also support sustainable manufacture by assisting companies in assessing and improving the circularity of their products, services and systems. On this basis, guidelines are given in the following sub-section to conduct this kind of workshop both for (i) educational purposes (e.g., on a training sequence related to circular economy or sustainable design), and (ii) industrial applications (e.g., to train engineers on circular economy principles or on managerial aspects to evaluate and monitor their circular economy strategies). 


\section{CONCLUSION: IMPLICATIONS AND PERSPECTIVES}

\subsection{Replicability and adaptation of the workshop}

Motivated by the aforementioned feedback, we intend to provide a turnkey, flexible and reusable workshop. The key guidelines to reproduce such a workshop are detailed below and, as aforementioned, all the resources needed are available on demand or in Saidani's PhD thesis (2018). One can adapt the proposed organisation (e.g., number of groups, C-indicators experimented) regarding the audience (e.g., training sequence for students, continuing education for engineers) and the time available to best fit his or her needs. Note that the workshop has been designed to be timeefficient, and the half-day format (see the suggested agenda and distribution of activities in Table 1) fits particularity with industrial constraints in time when it comes to conduct workshops on sustainable design or eco-innovation. Regarding the audience of such a workshop, it can be open to everyone (i.e., with a novice, intermediate or expert level) interested in circular design and life cycle thinking, in order to discover the possible contributions of circularity indicators and how they can be used in practice. Indeed, it is not necessary to be an expert or to have prior experience with circular economy practices or knowledge to use these $\mathrm{C}$-indicators and associated tools. Regarding the main aim of conducting such a workshop, it can help guiding designers, engineers, researchers, managers, administrators, decision-makers, or even policy-makers (e.g., using other C-indicators at a macro level of circular economy implementation) in identifying and selecting the most suitable(s) tool(s)/indicator(s) to assess, improve and/or monitor their circular strategies according to their specific needs and requirements. For instance, using such tools, designers would be able to integrate more easily circular thinking, as well as to evaluate the potential circularity performance of their ideas. Regarding the experimentation of $\mathrm{C}$-indicators, participants may have two possible options: (i) participants may test and experience the selection tool on their own projects to find out which circularity indicators suit the best to their needs. It can be possible for them to work individually or by group on their own project or specific case study linked to the circular economy. In this case, they will feed the query tool with their requirements so as to identify indicators that might be relevant for them. Then, as web access links are provided to explore the recommended indicators in output, attendees are free to test such circularity indicators; (ii) participants who do not have a particular case study or sufficient data to experience the tool by their own, but who are interested in testing the query tool and recommended indicators, it is possible to operate the data from the catalytic converter example to do so.

\subsection{Future research directions on circularity indicators}

Being able to forecast the circularity performance of a given product during design and development phases is a challenging task given the uncertainties of our complex and dynamic environment. Nevertheless, having the right tools to evaluate the circularity potential of industrial products with accuracy and in a robust manner is of the utmost importance in order to design effectively more circular artefacts that can affect our future in a sustainable way. This resonates particularly with the theme and discussion topics of the 22nd International Conference on Engineering Design (ICED19), namely 'Responsible Design for our Future'. Thanks to the experimentation of C-indicators through two workshops on the same industrial product, this study analyzed, compared and discussed the ability of four existing indicators to perform such tasks, i.e., their ability (i) at evaluating consistently the circularity potential of industrial products, and (ii) at providing sound orientations for improvement during design and development phases.

Against this background, key areas of progress for an augmented measurement and monitoring of the circular economy performance have been identified, with the aim to guide the future development and implementation of ad hoc circularity indicators. Indeed, the variability in the circularity scores provided by different potential C-indicators applied on the same product raises several questions. As a consequence, future works should incorporate uncertainty considerations in the assessment methodology of such indicators in order to assign, if any, a degree of incertitude to the circularity score for better soundness and acceptability. Also, the correlation between potential circularity indicators (e.g., the CEIP, CET, CPI) and effective circularity indicators (e.g., the MCI) is a line of research that needs to be further investigated, taking into account the time gap between a potential performance of circularity assessed during the design stage and the actual circularity performance at the end-of-life stage. In addition to the need to fine-tune the circularity scores through more advanced, 
justified and transparent scoring system, two other challenges need to be further addressed: the uptake of C-indicators by industry (e.g., by studying and facilitating their systematic integration into the design and development process of products); the relationship between an improvement in a circularity score and its consequences on different sustainability indicators (Walker et al., 2018), so as to consider possible rebound effects.

\section{REFERENCES}

Blessing, L. and Chakrabarti, A. (2009), DRM, a Design Research Methodology, Springer-Verlag London Limited.

Bonet, D., Petit, I. and Lancini, A. (2014), "L'économie circulaire : quelles mesures de la performance économique, environnementale et sociale?", Revue française de gestion industrielle, Vol. 33 No. 4.

Cayzer, S., Griffiths, P. and Beghetto, V. (2017), "Design of indicators for measuring product performance in the circular economy”, International Journal of Sustainable Engineering, Vol. 10, pp. 289-298.

Earley, R. (2017), “Circular Design Futures”, The Design Journal, Vol. 20 No. 4, pp. 421-434.

EASAC-European Academies Science Advisory Council (2016), Indicators for a Circular Economy, EASAC Policy Report No. 30, Halle, Germany.

EEA-European Environment Agency (2016), Circular economy in Europe - Developing the knowledge base, EEA Report No. 2/2016.

EMF-Ellen MacArthur Foundation (2015), Circularity Indicators - An Approach to Measure Circularity. Methodology \& Project Overview, Cowes, UK.

Evans, J. and Bocken, N. (2013), The Circular Economy Toolkit. Available online: http://circulareconomytoolkit.org (accessed 1 November 2018).

Eyckmans, J., García-Barragán, J.-F. and Rousseau, S. (2018), "Defining and measuring the circular economy: A mathematical approach", IECOMAT (Integrated economic modeling of material flows) Project, Pre-print, March 2018.

Geissdoerfer, M., Savaget, P., Bocken, N. and Hultink, E. (2017), “The Circular Economy - A new sustainability paradigm?”, Journal of Cleaner Production, Vol. 143, pp. 757-768.

Griffiths, P. and Cayzer, S. (2016), "Design of indicators for measuring product performance in the circular economy", International Conference on Sustainable Design and Manufacturing (SDM 2016), pp. 307-321.

Leroy, Y., Tyl, B., Vallet, F. and Cluzel, F. (2015), "Environmental evaluation of ideas in early phases: a challenging issue for design teams", International Conference on Engineering Design (ICED 15), Milan, Italy, Jul. 2015.

Linder, M., Sarasini, S. and van Loon, P. (2017), “A Metric for Quantifying Product-Level Circularity”, Journal of Industrial Ecology, Vol. 21, pp. 545-558.

OECD-Organisation for Economic Co-operation and Development (November 2014), Measuring and managing results in development co-operation, Report.

Parchomenko, A., Nelen, D., Gillabel, J. and Rechberger, H. (2019), "Measuring the circular economy - A Multiple Correspondence Analysis of 63 metrics", Journal of Cleaner Production, Vol. 210, pp. 200-216.

Saidani, M. (2018), Monitoring and advancing the circular economy transition - Circularity indicators and tools applied to the heavy vehicle industry, PhD Thesis, Université Paris-Saclay.

Saidani, M., Yannou, B., Leroy, Y. and Cluzel, F. (2017a), "Hybrid top-down and bottom-up framework to measure products' circularity performance", International Conference on Engineering Design (ICED 17), Vancouver, Canada, Aug. 2017.

Saidani, M., Yannou, B., Leroy, Y. and Cluzel, F. (2017b), "How to Assess Product Performance in the Circular Economy? Proposed Requirements for the Design of a Circularity Measurement Framework", Recycling, Vol. 2 No. 6.

Saidani, M., Yannou, B., Leroy, Y., Cluzel, F. and Kendall, A. (2019), “A taxonomy of circular economy indicators", Journal of Cleaner Production, Vol. 207, pp. 542-559.

Walker, S., Coleman, N., Hodgson, P., Collins, N. and Brimacombe, L. (2018), "Evaluating the Environmental Dimension of Material Efficiency Strategies Relating to the Circular Economy", Sustainability, Vol. 10 No. 3, pp. 666-680.

\section{ACKNOWLEDGMENTS}

The workshops, from which the results are analyzed and discussed in this research paper, were carried out in the context of the doctoral spring school "Eco-design of complex system", funded by EcoSD and S-mart, and endorsed by the Design Society. The authors would like to thank the participants of the 2017 and 2018 editions of this spring school for their insightful contributions. 